\title{
Distributed Fault Estimation of Nonlinear Networked Systems: Application to Robotic Manipulator
}

\author{
Juntong Chen, ${ }^{1,2}$ Rongyao Ling, ${ }^{1}$ Hongjie Ni, ${ }^{1}$ and Dan Zhang ${ }^{1}$ \\ ${ }^{1}$ Department of Automation, Zhejiang University of Technology, Hangzhou 310032, China \\ ${ }^{2}$ School of Automation and Electrical and Engineering, Zhejiang University of Science and Technology, Hangzhou 310032, China \\ Correspondence should be addressed to Dan Zhang; jason_zhang19850626@hotmail.com
}

Received 10 June 2014; Accepted 15 July 2014; Published 12 August 2014

Academic Editor: Yun-Bo Zhao

Copyright (c) 2014 Juntong Chen et al. This is an open access article distributed under the Creative Commons Attribution License, which permits unrestricted use, distribution, and reproduction in any medium, provided the original work is properly cited.

\begin{abstract}
This paper is concerned with the distributed fault estimation for a class of nonlinear networked systems, where the T-S fuzzy model is utilized to approximate the nonlinear plant and the whole fault estimation task is operated by a wireless sensor network. Due to the limited power in sensors, signal is quantized before transmission. Based on the Lyapunov stability theory and the robust control approach, a sufficient condition is obtained such that the estimation error system is asymptotic stable with a prescribed $H_{\infty}$ performance level. Finally, a case study on the actuator fault estimation of robotic manipulator is given to show the effectiveness of the proposed design.
\end{abstract}

\section{Introduction}

Fault detection and isolation (FDI) and fault tolerant control (FTC) have received considerable attention in the past two decades due to the increasing demand for higher performance, higher safety, and reliability standards in engineering. Till now, many effective FDI methods have been proposed including the model-based fault detection approach [1], parity relations approach [2], the Kalman filter-based approach [3], and so on. Based on the FDI information, the controller is reconstructed to compensate the fault, which can guarantee the stability of systems and also some certain performance $[4$, 5]. For example, the authors in [6] proposed a methodology for detection and accommodation of actuator faults for a class of multi-input-multi-output (MIMO) stochastic systems. Firstly, a new real-time fault estimation module that estimates the actuator effectiveness was developed. Then, the output of the nominal controller is reconfigured to compensate for the loss of actuator effectiveness in the system. Simulation results of a helicopter in vertical plane were finally presented to demonstrate the performance of the proposed fault-tolerant control scheme. In [7], the authors studied the problem of robust fault estimation (FE) observer design for discrete-time Takagi-Sugeno (T-S) fuzzy systems via piecewise Lyapunov functions. Both the full-order FE observer (FFEO) and the reduced-order FE observer (RFEO) were presented. They showed that the optimal fault estimator can be determined by solving a set of linear matrix inequalities. It should be noted that all the above fault estimators are designed in a centralized way, and such a framework may have low reliability; for example, the estimation task may fail once the estimator has unrecoverable fault.

With the development of wireless sensor networks (WSNs), distributed estimation has received much attention in the last decade. Compared with the centralized filtering systems, the distributed filtering one has more redundancies, and thus it has higher reliability. Once some sensors have unrecoverable fault, other sensors can also provide the estimation signal. For example, a distributed Kalman filtering algorithm has been introduced in [8] that allows the nodes of a sensor network to track the average of $n$ sensor measurements using an average consensus based distributed filter. In the scenario that the priori information on the external noises is not precisely known, the authors in [9] proposed an average $H_{\infty}$ performance and the distributed observer design has been presented such that the prescribed estimation performance is guaranteed in the $H_{\infty}$ sense. Recently, the 
distributed filtering for a class of sensor networks with switching topology is investigated in our earlier work [10]. Based on the switched system approach, we showed that the sensor working mode can be regulated to save energy. Recent advancement on the distributed filtering in sensor networks is referred to [8-10].

It is worth pointing out that the above distributed filtering results only focused on the state estimation problem. However, certain faults may occur in practical systems as we have mentioned above. In particular, in today's industrial systems, actuator fault may occur due to the increasing complexity of systems. Then, a natural problem is how to estimate the fault in a distributed way? To the best of the author's knowledge, such a challenging work has not been investigated yet. In this paper, we are concerned with the distributed fault estimation for a class of nonlinear systems. Two fundamental difficulties are identified as follows: (1) in the sensor-network-based distributed fault estimation systems, the first difficulty is how to prolong the lifetime of the networks as the sensor power is usually limited and it is impossible to be replaced when deployed in a large geometric area. (2) For a sensor network, each filter is designed based on the local information and the information from its neighboring ones, so the second difficulty is how to handle the complicated couplings between one sensor and its neighboring sensors in the presence of multiple quantization errors.

To handle the above challenges, attention of this paper is focused on designing a set of distributed fault estimators such that the estimation error system is asymptotically stable and achieves a prescribed $H_{\infty}$ performance. Firstly, the Kronecker product is introduced to help solve the complex coupling of sensors in network. Then, signal quantization technique is utilized to reduce the transmitted packet size and thus save the transmission power. Based on the robust control technique and the Lyapunov stability theory, a new sufficient condition is obtained for the solvability of the considered estimation problem. Finally, a case study of robotic manipulator is given to show the effectiveness of the proposed design.

Notation. Some definitions for the notation used throughout the paper are given as follows. A superscript $\mathrm{T}$ stands for matrix transposition and superscript -1 stands for the inverse of a matrix; $\mathbb{R}^{n}$ denotes the $n$-dimensional Euclidean space and $\|\cdot\|$ denotes the Euclidean norm; $l_{2}[0, \infty)$ denotes the space of square-integrable vector functions over $[0, \infty)$ and the symbol $\otimes$ denotes the Kronecker product; $I$ and 0 represent the identity matrix and zero matrix with appropriate dimensions; $e_{n}$ represents an $n$-dimensional column vector with all the entries being identity.

\section{Problem Formulation}

The sensor network is deployed to monitor the plant, where there is no centralized fusion center in the network, and every sensor in the network acts also as an estimator.

Standard definitions for the sensor networks are now given as follows. Let the topology of a given sensor network be represented by a direct graph $\pi(k)=(\vartheta, \chi, A)$ of order $n$ with the set of sensors $\vartheta=\{1,2, \ldots, n\}$, set of edges $\chi \subseteq \vartheta \times \vartheta$, and a weighted adjacency matrix $A=\left[a_{i j}\right]$ with nonnegative adjacency elements $a_{i j}$. An edge of $\pi$ is denoted by $(i, j)$. The adjacency elements associated with the edges of the graph are $a_{i j}=1,(i, j) \in \vartheta$, if sensor $i$ receives information from sensor $j$, whereas $a_{i j}=0$, if sensor $i$ can not receive information from sensor $j$. Moreover, we assume $a_{i i}=1$ for all $i \in \vartheta$. The set of neighbors of node $i \in \vartheta$ plus the node itself are denoted by $N_{i}=\{j \in \delta:(i, j) \in \vartheta\} . A=\left[a_{i j}\right]$ is a square matrix representing the topology of the sensor network.

In this paper, the nonlinear plant is described by the following T-S model.

Plant Rule i. IF $\phi_{1}(k)$ is $\psi_{i 1}$ and $\phi_{2}(k)$ is $\psi_{i 2}$ and $\cdots$ and $\phi_{t}(k)$ is $\psi_{i t}$, THEN

$$
\begin{gathered}
x(k+1)=A_{i} x(k)+B_{i} u(k)+D_{i} w(k)+E_{i} f(k) \\
y_{p}(k)=C_{p i} x(k)+D_{p i} w(k), \quad i=1,2, \ldots, r,
\end{gathered}
$$

where $\phi(k)=\left[\phi_{1}(k), \phi_{2}(k), \ldots, \phi_{t}(k)\right]$ is the premise variable vector, $\psi_{i j}$ is the fuzzy set, and $r$ is the number of IF-THEN rules. $x(t) \in \mathbb{R}^{x}$ is the state variable, $u(k) \in \mathbb{R}^{u}$ is the input, $w(k) \in \mathbb{R}^{w}$ is the unknown disturbance belonging to $l_{2}[0, \infty)$, $f(k) \in \mathbb{R}^{f}$ is the fault vector, $y_{p}(k) \in \mathbb{R}^{y_{p}} \quad(p=1,2, \ldots, n)$ is the output measured by the $p$ th fault estimator, and $A_{i}, B_{i}$, $D_{i}, E_{i}, C_{p i}, D_{p i}$ are all known real matrices with appropriate dimensions.

Remark 1. It should be pointed out that the T-S fuzzy model (1) can only approximate the smooth nonlinear control systems, but not all the nonlinear control systems. Our attention in this paper is to design the fault estimator for such a class of nonlinear systems described by the T-S fuzzy model. We will show in the simulation part that such a $\mathrm{T}$ $S$ fuzzy system can be used to model the nonlinear robotic manipulator.

By using a center-average defuzzifier, product fuzzy inference, and a singleton fuzzifier, the overall fuzzy system is inferred as

$$
\begin{aligned}
x(k+1)= & \sum_{i=1}^{r} h_{i}(\phi(k)) \\
& \times\left[A_{i} x(k)+B_{i} u(k)+D_{i} w(k)+E_{i} f(k)\right] \\
y_{p}(k)= & \sum_{i=1}^{r} h_{i}(\phi(k))\left[C_{p i} x(k)+D_{p i} w(k)\right],
\end{aligned}
$$

where $h_{i}(\phi(k))=\pi_{i}(\phi(k)) / \sum_{i=1}^{r} \pi_{i}(\phi(k))$ and $\pi_{i}(\phi(k))=$ $\prod_{j=1}^{p} \psi_{i j}\left(\phi_{j}(k)\right)$, with $\psi_{i j}\left(\phi_{j}(k)\right)$ representing the grade of membership of $\phi_{j}(k)$ in $\psi_{i j}$. Usually, it is assumed that $\pi_{i}((\phi(k))) \geq 0$ and $\sum_{i=1}^{r} \pi_{i}(\phi(k))>0$ for all $\phi(k)$. 
For system (2), the following distributed fault estimator form is constructed.

Plant Rule i. IF $\phi_{1}(k)$ is $\psi_{i 1}$ and $\phi_{2}(k)$ is $\psi_{i 2}$ and $\cdots$ and $\phi_{t}(k)$ is $\psi_{i t}$, THEN

$$
\begin{aligned}
\widehat{x}_{p}(k+1)= & A_{i} x_{p}(k)+B_{i} u(k) \\
& +E_{i} \widehat{f}_{p}(k)-\sum_{q=1}^{n} a_{p q} H_{p q}^{i} Q_{q}\left(\widehat{y}_{q}(k)-y_{q}(k)\right), \\
\widehat{y}_{p}(k)= & C_{p i} \widehat{x}_{p}(k), \\
\widehat{f}_{p}(k+1)= & \widehat{f}_{p}(k)-\sum_{q=1}^{n} a_{p q} K_{p q}^{i} Q_{q}\left(\widehat{y}_{q}(k)-y_{q}(k)\right),
\end{aligned}
$$

where $\widehat{x}_{p} \in \mathbb{R}^{x}$ is the estimator state vector, $\hat{y}_{p}(k) \in \mathbb{R}^{y_{p}}(p=$ $1,2, \ldots, n)$ is the estimator output, and $\widehat{f}_{p}(k) \in \mathbb{R}^{f}$ is an estimate of $f(k) ; H_{p q}^{i}$ and $K_{p q}^{i}$ are the estimator gain matrices to be designed. $Q_{q}(\cdot)$ represents the quantizer in $p$ th estimator with the quantization density $0<\rho_{p}<1$ and the set of quantization levels are described as

$$
\begin{array}{r}
U_{j}=\left\{ \pm u_{i}^{j}: u_{i+1}^{j}=\rho_{j} u_{i}^{j}, i=1,2,3 \cdots\right\} \cup\left\{ \pm u_{0}^{j}\right\} \cup\{0\} \\
0<\rho_{j}<1, u_{0}^{j}>0 .
\end{array}
$$

Its quantization is described by

$$
Q_{q}(\sigma)=\left[\begin{array}{lllll}
Q_{q}\left(\sigma_{1}\right) & Q_{q}\left(\sigma_{2}\right) & \cdots & Q_{q}\left(\sigma_{N}\right)
\end{array}\right]^{\mathrm{T}}
$$

Here, $\sigma=\left[\sigma_{1}, \sigma_{2}, \ldots, \sigma_{N}\right]$ is the input vector; the quantized output $Q_{q}\left(\sigma_{k}\right)$ is given by the following piecewise function

$$
Q_{q}\left(\sigma_{k}\right)= \begin{cases}\rho_{q}^{i} u_{0}^{q}, & \text { if } \frac{u_{i}^{q}}{1+\delta_{q}}<\sigma_{k} \leq \frac{u_{i}^{q}}{1-\delta_{q}}, \sigma_{k}>0 \\ 0, & \text { if } \sigma_{k}=0 \\ -Q_{q}\left(-\sigma_{k}\right), & \text { if } \sigma_{k}<0,\end{cases}
$$

where $\delta_{q}=\left(1-\rho_{q}\right) /\left(1+\rho_{q}\right)$ is the maximum error coefficient of quantizer $Q_{q}(\cdot)$. In this paper, the quantization error is defined as

$$
e_{\mathrm{Q}_{q}}=Q_{q}\left(\sigma_{k}\right)-\sigma_{k}=\Delta_{q} \sigma_{k}, \quad \Delta_{q} \in\left[-\delta_{q}, \delta_{q}\right]
$$

Let $\Delta f(k)=f(k+1)-f(k)$, and let the tracking errors of $x$ and $f$ be

$$
\begin{aligned}
& e_{x_{p}}(k)=\widehat{x}_{p}(k)-x(k), \\
& e_{f_{p}}(k)=\widehat{f}_{p}(k)-f(k) .
\end{aligned}
$$

Then, based on (1) and (3), the error dynamics can be derived as

$$
\begin{aligned}
e_{x_{p}}(k+1)=\sum_{i=1}^{r} & \sum_{j=1}^{r} h_{i}(\phi(k)) h_{j}(\phi(k)) \\
& \times\left[A_{i} e_{x_{p}}(k)+E_{i} e_{f_{p}}(k)\right. \\
& -\sum_{q=1}^{n} a_{p q} H_{p q}^{i}\left(I+\bar{\Delta}_{q}(k)\right) \\
& \left.\times\left(C_{q j} e_{x_{p}}(k)-D_{q j} w(k)\right)-D_{i} w(k)\right]
\end{aligned}
$$

$$
\begin{aligned}
e_{f_{p}}(k+1)=\sum_{i=1}^{r} \sum_{j=1}^{r} h_{i}(\phi(k)) h_{j}(\phi(k)) & \\
\times & {\left[e_{f_{p}}(k)-\Delta f(k)\right.} \\
& -\sum_{q=1}^{n} a_{p q} K_{p q}^{i}\left(I+\bar{\Delta}_{q}(k)\right) \\
& \left.\times\left(C_{q j} e_{x_{p}}(k)-D_{q j} w(k)\right)\right],
\end{aligned}
$$

where $\bar{\Delta}_{q}(k)=\operatorname{diag}\left\{\Delta_{q 1}(k), \Delta_{q 2}(k), \ldots, \Delta_{q y}(k)\right\}$, and $\Delta_{q i} \in$ $\left[-\delta_{q}, \delta_{q}\right], i=1,2, \ldots, y$.

$$
\text { Let }
$$

$$
\begin{array}{ll}
\bar{e}_{x}(k)=\left[\begin{array}{lll}
e_{x_{1}}^{\mathrm{T}}(k) & \cdots & e_{x_{n}}^{\mathrm{T}}(k)
\end{array}\right]^{\mathrm{T}}, & \bar{A}_{i}=I_{n} \otimes A_{i}, \\
\bar{e}_{f}(k)=\left[\begin{array}{llll}
e_{f_{1}}^{\mathrm{T}}(k) & \cdots & e_{f_{n}}^{\mathrm{T}}(k)
\end{array}\right]^{\mathrm{T}}, & \bar{E}_{i}=I_{n} \otimes E_{i},
\end{array}
$$

$$
\bar{C}_{i}=\operatorname{diag}\left\{C_{1 i}, C_{2 i} \cdots C_{n i}\right\} \text {, }
$$

$$
\begin{gathered}
\bar{D}_{i}=\left[\begin{array}{lll}
D_{1 i}^{\mathrm{T}} & \cdots & D_{n i}^{\mathrm{T}}
\end{array}\right]^{\mathrm{T}}, \quad \widetilde{D}_{i}=e_{n} \otimes D_{i}, \\
\Delta(k)=\operatorname{diag}\left\{\bar{\Delta}_{1}(k), \bar{\Delta}_{2}(k) \cdots \bar{\Delta}_{n}(k)\right\},
\end{gathered}
$$

$$
\begin{gathered}
\bar{H}_{i}=\left[\begin{array}{ccc}
a_{11} H_{11}^{i} & \cdots & a_{1 n} H_{1 n}^{i} \\
\vdots & \ddots & \vdots \\
a_{n 1} H_{n 1}^{i} & \cdots & a_{n n} H_{n n}^{i}
\end{array}\right], \\
\bar{K}_{i}=\left[\begin{array}{ccc}
a_{11} K_{11}^{i} & \cdots & a_{1 n} K_{1 n}^{i} \\
\vdots & \ddots & \vdots \\
a_{n 1} K_{n 1}^{i} & \cdots & a_{n n} K_{n n}^{i}
\end{array}\right] .
\end{gathered}
$$


By defining $h_{i}^{k}=h_{i}(\phi(k)), \bar{e}(k)=\left[\begin{array}{ll}\bar{e}_{x}^{\mathrm{T}}(k) & \bar{e}_{f}^{\mathrm{T}}(k)\end{array}\right]^{\mathrm{T}}, v(k)=$ $\left[w^{\mathrm{T}}(k) \Delta f^{\mathrm{T}}(k)\right]^{\mathrm{T}}$, combining (9) and (10), the following augmented estimation error system is obtained:

$$
\begin{gathered}
\bar{e}(k+1)=\sum_{i=1}^{r} \sum_{j=1}^{r} h_{i}^{k} h_{j}^{k}\left[\bar{M}_{i j} \bar{e}(k)+\bar{N}_{i j} v(k)\right], \\
z(k)=L \bar{e}(k),
\end{gathered}
$$

where

$$
\begin{gathered}
\bar{M}_{i j}=\left[\begin{array}{cc}
\bar{A}_{i}-\bar{H}_{i}(I+\Delta(k)) \bar{C}_{j} & \bar{E}_{i} \\
-\bar{K}_{i}(I+\Delta(k)) \bar{C}_{j} & I
\end{array}\right], \\
\bar{N}_{i j}=\left[\begin{array}{cc}
\bar{H}_{i}(I+\Delta(k)) \bar{D}_{j}-\widetilde{D}_{i} & 0 \\
\bar{K}_{i}(I+\Delta(k)) \bar{D}_{j} & e_{n} \otimes I
\end{array}\right], \\
L=\left[\begin{array}{ll}
0 & I
\end{array}\right] .
\end{gathered}
$$

The objective of this paper is to design the robust distributed fault estimator in the form of (3) such that the estimation error system (12) is asymptotic stable and achieves an $H_{\infty}$ performance. To be more specific, the estimation requirements are expressed as follows.

(1) The augmented estimation error system in (12) with $v(k) \equiv 0$ is asymptotically stable.

(2) For any nonzero external disturbance $v(k) \in l_{2}[0, \infty)$, its effect on the estimation error $e_{f_{p}}(k)$ is attenuated below a desired level $\gamma>0$. More specifically, it is required that

$$
\sum_{k=0}^{\infty} \frac{1}{n}\|z(k)\|^{2} \leq \gamma^{2} \sum_{k=0}^{\infty}\|v(k)\|^{2} .
$$

The estimation error system (12) is said to be asymptotically stable with an average $H_{\infty}$ performance $\gamma$ if the aforementioned requirements are met.

Remark 2. In this paper, our attention is focused on estimating the fault signal in the presence of unknown disturbance. Hence, we choose $L=\left[\begin{array}{ll}0 & I\end{array}\right]$. It is interesting to see that when we choose $L$ as an identity matrix, both state and fault signals can be estimated such that the estimation error system is asymptotic stable and achieves a prescribed $H_{\infty}$ performance level.

The following lemmas are introduced before further proceeding.

Lemma 3 (see [11]). For given matrices of appropriate dimensions $\Sigma_{1}, \Sigma_{2}$, and $\Sigma_{3}$ with $\Sigma_{1}$ satisfying $\Sigma_{1}=\Sigma_{1}^{T}$, then

$$
\Sigma_{1}+\Sigma_{2} \Delta \Sigma_{3}+\Sigma_{3}^{T} \Delta^{T} \Sigma_{2}^{T}<0
$$

holds for all $\Delta^{T} \Delta \leq I$ if and only if there exists a scalar $\varepsilon>0$ such that

$$
\Sigma_{1}+\varepsilon \Sigma_{2} \Sigma_{2}^{T}+\varepsilon^{-1} \Sigma_{3}^{T} \Sigma_{3}<0
$$

Lemma 4 (see [12]). For matrices $A, Q=Q^{T}$, and $P>0$ the following matrix inequality

$$
A^{T} P A-Q<0
$$

holds if and only if there exists a matrix $T$ of appropriate dimensions such that

$$
\left[\begin{array}{cc}
-Q & A^{T} T \\
* & P-T-T^{T}
\end{array}\right]<0 .
$$

\section{Main Results}

In this section, we aim to solve the distributed fault estimation problem. Some theorems are derived that the estimation error system (12) is asymptotic stable and achieves an $H_{\infty}$ performance. Theorem 5 guarantees that the estimation error system (12) is asymptotic stable with an $H_{\infty}$ performance $\gamma>0$, and the specific design method for distributed fault estimator is proposed in Theorem 7 .

Theorem 5. For a given system (3) and a scalar $\gamma>0$, the estimation error system (12) is asymptotic stable with an $H_{\infty}$ performance $\gamma>0$ if there exist symmetric positive matrices $P$ and a scalar $\varepsilon>0$, the following inequalities

$$
\left[\begin{array}{cccccc}
-P & 0 & \widehat{M}_{i j}^{T} & L^{T} & \varepsilon \widehat{C}_{j}^{T} \Delta & 0 \\
* & -\gamma^{2} I & \widehat{N}_{i j}^{T} & 0 & \varepsilon \widehat{D}_{j}^{T} \Delta & 0 \\
* & * & -P^{-1} & 0 & 0 & F_{i} \\
* & * & * & -n I & 0 & 0 \\
* & * & * & * & -\varepsilon I & 0 \\
* & * & * & * & * & -\varepsilon I
\end{array}\right]<0
$$

hold for all $i, j=1,2, \ldots, r$, where

$$
\begin{gathered}
\widehat{M}_{i j}=\left[\begin{array}{cc}
\bar{A}_{i}-\bar{H}_{i} \bar{C}_{j} & \bar{E}_{i} \\
-\bar{K}_{i} \bar{C}_{j} & I
\end{array}\right], \quad \widehat{C}_{j}=\left[\begin{array}{ll}
-\bar{C}_{j} & 0
\end{array}\right], \\
\widehat{N}_{i j}=\left[\begin{array}{cc}
\bar{H}_{i} \bar{D}_{j}-\widetilde{D}_{i} & 0 \\
\bar{K}_{i} \bar{D}_{j} & e_{n} \otimes I
\end{array}\right], \quad \widehat{D}_{j}=\left[\begin{array}{ll}
\bar{D}_{j} & 0
\end{array}\right], \\
F_{i}=\left[\bar{H}_{i}^{T} \bar{K}_{i}^{T}\right]^{T}, \quad \Delta=\operatorname{diag}\left\{\delta_{1} I_{y}, \delta_{2} I_{y}, \ldots, \delta_{n} I_{y}\right\} .
\end{gathered}
$$

Proof. We first consider the asymptotic stability of the augmented estimation error system (12) with $v(k) \equiv 0$. Construct the following Lyapunov function:

$$
V(k)=\bar{e}^{\mathrm{T}}(k) P \bar{e}(k) ;
$$

then the dynamics of $V(k)$ is given by

$$
\begin{aligned}
\Delta V(k) & \triangleq V(k+1)-V(k) \\
& =\sum_{i=1}^{r} \sum_{j=1}^{r} h_{i}^{k} h_{j}^{k}\left[\bar{e}^{\mathrm{T}}(k+1) P \bar{e}(k+1)-\bar{e}^{\mathrm{T}}(k) P \bar{e}(k)\right] \\
& =\sum_{i=1}^{r} \sum_{j=1}^{r} h_{i}^{k} h_{j}^{k}\left[\bar{e}^{\mathrm{T}}(k)\left(\bar{M}_{i j}^{\mathrm{T}} P \bar{M}_{i j}-P\right) \bar{e}(k)\right]
\end{aligned}
$$


where $\bar{M}_{i j}=M_{i}+F_{i} \widehat{C}_{j}+F_{i} \Delta(k) \widehat{C}_{j}$ and $M_{i}=\left[\begin{array}{cc}\bar{A}_{i} & \bar{E}_{i} \\ 0 & I\end{array}\right], N_{i}=$ $\left[\begin{array}{cc}-\widetilde{D}_{i} & 0 \\ 0 & e_{n} \otimes I\end{array}\right]$. It can be obtained that $\Delta V(k)=$ $\sum_{i=1}^{r} \sum_{j=1}^{r} h_{i}^{k} h_{j}^{k}\left[\Omega_{i j}\right]$, where $\Omega_{i j}=\Omega_{1 i}+\Omega_{2 j} \Delta(k) \Omega_{3 i}+$ $\Omega_{3 i}^{\mathrm{T}} \Delta^{\mathrm{T}}(k) \Omega_{2 j}^{\mathrm{T}}, \Omega_{1 i}=\left[\begin{array}{cc}-P & \widehat{M}_{i}^{\mathrm{T}} \\ * & -P^{-1}\end{array}\right], \Omega_{2 j}=\left[\begin{array}{ll}\widehat{C}_{j} & 0\end{array}\right]^{\mathrm{T}}, \Omega_{3 i}=$ $\left[\begin{array}{ll}0 & F_{i}^{\mathrm{T}}\end{array}\right]$.

Notice that $\left(\Delta(k) \Delta^{-1}\right)^{\mathrm{T}} \Delta(k) \Delta^{-1} \leq I$; by using Lemma 3 and Schur complement, it can be seen that $\Omega_{i j}<0$ is equivalent to

$$
\left[\begin{array}{cccc}
-P & \widehat{M}_{i j}^{\mathrm{T}} & \varepsilon \widehat{C}_{j}^{\mathrm{T}} \Delta & 0 \\
* & -P^{-1} & 0 & F_{i} \\
* & * & -\varepsilon I & 0 \\
* & * & * & -\varepsilon I
\end{array}\right]<0, \quad i, j=1,2, \ldots, r .
$$

It is noted that (23) holds if (19) holds; hence we have $\Delta V(k)<0$, indicating that the estimation error system (12) is asymptotic stable.

We then consider the $H_{\infty}$ performance of the estimation error system (12). Define

$$
J \triangleq \sum_{k=0}^{\infty}\left[\frac{1}{n} z^{\mathrm{T}}(k) z(k)-\gamma^{2} v^{\mathrm{T}}(k) v(k)\right] .
$$

Denote $\eta(k)=\left[\begin{array}{lll}\bar{e}^{\mathrm{T}}(k) & v^{\mathrm{T}}(k)\end{array}\right]^{\mathrm{T}}$; then it can be obtained under the zero initial conditions that

$$
\begin{aligned}
J & \leq \sum_{k=0}^{\infty}\left[\frac{1}{n} z^{\mathrm{T}}(k) z(k)-\gamma^{2} v^{\mathrm{T}}(k) v(k)+\Delta V\right] \\
& =\sum_{k=0}^{\infty} \sum_{i=1}^{r} \sum_{j=1}^{r} h_{i}^{k} h_{j}^{k}\left[\eta^{\mathrm{T}}(k) \Theta_{i j} \eta(k)\right],
\end{aligned}
$$

where $\Theta_{i j}=\Theta_{1 i}+\Theta_{2 j} \Delta(k) \Theta_{3 i}+\Theta_{3 i}^{\mathrm{T}} \Delta^{\mathrm{T}}(k) \Theta_{2 j}^{\mathrm{T}}$ with

$$
\begin{aligned}
\Theta_{1 j} & =\left[\begin{array}{llll}
\widehat{C}_{j} & \widehat{D}_{j} & 0 & 0
\end{array}\right]^{\mathrm{T}}, \\
\Theta_{2 i} & =\left[\begin{array}{llll}
0 & 0 & F_{i}^{\mathrm{T}} & 0
\end{array}\right], \\
\Theta_{3 i} & =\left[\begin{array}{cccc}
-P & 0 & \widehat{M}_{i}^{\mathrm{T}} & L^{\mathrm{T}} \\
* & -\gamma^{2} I & \widehat{N}_{i}^{\mathrm{T}} & 0 \\
* & * & -P^{-1} & 0 \\
* & * & * & -n I
\end{array}\right] .
\end{aligned}
$$

Based on Lemma 3 and Schur complement, it can be derived from (19) that $\Theta_{i j}<0$. Thus $J<0$ and the estimation error system (12) is asymptotic stable with an $H_{\infty}$ performance $\gamma>0$. The proof is completed.

Remark 6. In Theorem 5, it is difficult to determine the estimator gains due to the nonlinear term in (19). Toward this end, based on Theorem 5, Theorem 7 is proposed to determine the estimator gains.

Theorem 7. For a given system (3) and a scalar $\gamma>0$, the estimation error system (12) is asymptotic stable with an average $H_{\infty}$ performance $\gamma>0$ if there exist symmetric positive matrices $P$, a matrix $T$, and a scalar $\varepsilon>0$; the following inequalities

$$
\left[\begin{array}{cccccc}
-P & 0 & M_{i}^{T} T+\widehat{C}_{j}^{T} \bar{F}_{i}^{T} & L^{T} & \varepsilon \widehat{C}_{j}^{T} \Delta & 0 \\
* & -\gamma^{2} I & N_{i}^{T} T+\widehat{D}_{j}^{T} \bar{F}_{i}^{T} & 0 & \varepsilon \widehat{D}_{j}^{T} \Delta & 0 \\
* & * & P-T-T^{T} & 0 & 0 & \bar{F}_{i} \\
* & * & * & -n I & 0 & 0 \\
* & * & * & * & -\varepsilon I & 0 \\
* & * & * & * & * & -\varepsilon I
\end{array}\right]<0
$$

hold for all $i, j=1,2, \ldots, r$. Meanwhile, the estimator gains can be determined as

$$
F_{i}=T^{-T} \bar{F}_{i}
$$

Proof. Based on Lemma 4 and Schur complement, it is easy to derive from (27) that (29) holds. Denote $\bar{T}=$ $\operatorname{diag}\left\{I, I, T^{-\mathrm{T}}, I, I, I\right\}$; premultiplying (28) by $\bar{T}$ and postmultiplying (29) by $\bar{T}^{\mathrm{T}}$ simultaneously, (18) is obtained, which ends the proof. Consider

$$
\left[\begin{array}{cccccc}
-P & 0 & M_{i}^{\mathrm{T}} T+\widehat{C}_{j}^{\mathrm{T}} \bar{F}_{i}^{\mathrm{T}} & L^{\mathrm{T}} & \varepsilon \widehat{C}_{j}^{\mathrm{T}} \Delta & 0 \\
* & -\gamma^{2} I & N_{i}^{\mathrm{T}} T+\widehat{D}_{j}^{\mathrm{T}} \bar{F}_{i}^{\mathrm{T}} & 0 & \varepsilon \widehat{D}_{j}^{\mathrm{T}} \Delta & 0 \\
* & * & T P^{-1} T^{\mathrm{T}} & 0 & 0 & \bar{F}_{i} \\
* & * & * & -n I & 0 & 0 \\
* & * & * & * & -\varepsilon I & 0 \\
* & * & * & * & * & -\varepsilon I
\end{array}\right]<0 .
$$

Remark 8. Optimal solutions for the problem of distributed fault estimation can be obtained by solving the following optimization problem:

$$
\begin{aligned}
& \min \gamma^{2} \\
& \text { s.t. } \quad(27) \text {. }
\end{aligned}
$$

The estimator gains can be determined through (28).

\section{Illustrative Example}

In this section, a simulation example is presented to illustrate the effectiveness of the proposed design methods. Consider a single-link rigid robot that is connected through a revolute joint to the basement and whose plane of motion is vertical. The motion equation of this mechanical system is given by $[13,14]$

$$
J \ddot{\vartheta}=-(0.5 m g l+M g l) \sin (\vartheta)+u,
$$

where $\vartheta$ denotes the joint rotation angle in radians, $m=1.5 \mathrm{~kg}$ is the mass of the load, $M=3 \mathrm{~kg}$ is the mass of the rigid link, $g=9.8 \mathrm{~m} / \mathrm{s}^{2}$ is the gravity constant, $l=0.5 \mathrm{~m}$ is the length of the robot link, $J=0.875 \mathrm{~kg} \cdot \mathrm{m}^{2}$ is the moment of inertia, and $u$ is the control torque applied at the joint in $\mathrm{Nm} . \vartheta=0$ denotes the lowest vertical equilibrium position. 


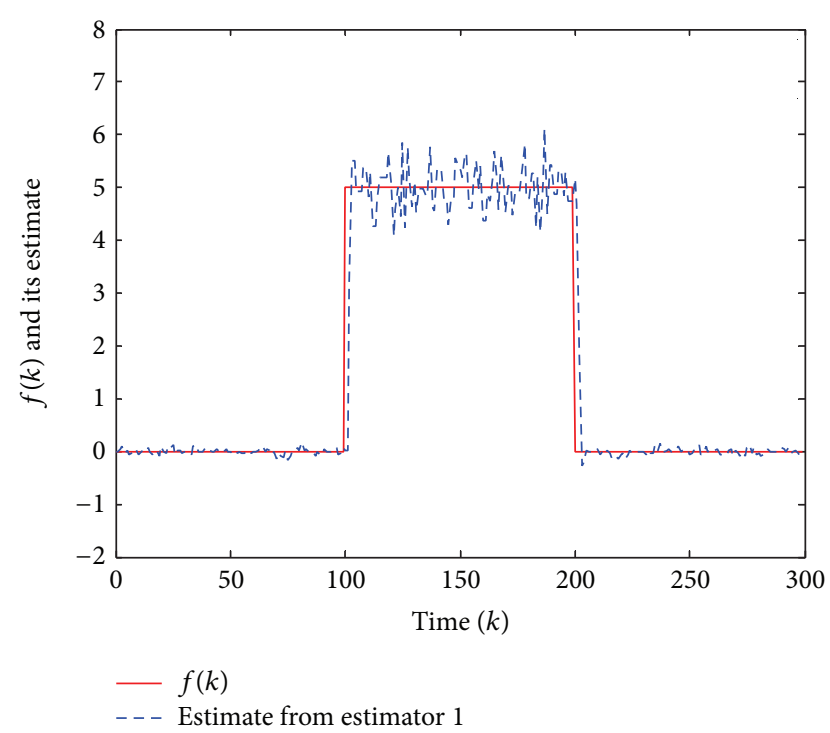

FIGURE 1: $f(k)$ and its estimate $f_{1}(k)$.

By applying the approach in [13], a T-S fuzzy model is constructed for system (31) as follows:

Plant rule 1: IF $x_{1}$ is about 0 , THEN

$$
\dot{x}=A_{1 c} x+B_{1} u+w
$$

Plant rule 2: IF $x_{1}$ is about $\Pi$, THEN

$$
\dot{x}=A_{2 c} x+B_{2} u+w,
$$

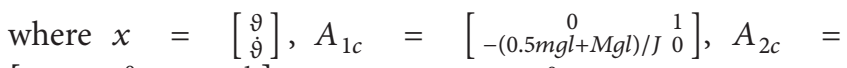
$\left[\begin{array}{cc}0 & 1 \\ -2(0.5 m g l+M g l) / \pi J & 0\end{array}\right]$, and $B_{1 c}=B_{2 c}=\left[\begin{array}{c}0 \\ 1 / J\end{array}\right]$.

The fuzzy membership functions are set as $h_{1}\left(x_{1}\right)=$ $\left(0.5 \pi-\left|x_{1}\right|\right) / 0.5 \pi$ and $h_{2}\left(x_{1}\right)=1-h_{1}\left(x_{1}\right)$. By using the controller gain $K_{1}=\left[\begin{array}{lll}10.5000 & -5.1057\end{array}\right], K_{2}=$ $\left[\begin{array}{ll}3.8800 & -5.0628\end{array}\right]$, with a sampling time $T_{s}=0.5 \mathrm{~s}$, the discrete-time model for system (32) is obtained as

Plant rule 1: IF $x_{1}(k)$ is about 0 , THEN

$$
x(k+1)=A_{1} x(k)+B_{1} w(k)
$$

Plant rule 2: IF $x_{1}(k)$ is about $\Pi$, THEN

$$
x(k+1)=A_{2} x(k)+B_{2} w(k),
$$

where

$$
\begin{array}{ll}
A_{1}=\left[\begin{array}{cc}
0.5508 & 0.1139 \\
-1.0252 & -0.1139
\end{array}\right], & B_{1}=\left[\begin{array}{l}
0.0570 \\
0.1302
\end{array}\right], \\
A_{2}=\left[\begin{array}{cc}
0.5515 & 0.1149 \\
-1.0270 & -0.1136
\end{array}\right], & B_{2}=\left[\begin{array}{l}
0.0574 \\
0.1314
\end{array}\right] .
\end{array}
$$

In this example, we consider the actuator fault problem, and we aim to estimate the fault in a distributed framework. Hence, $E_{i}=B_{i}$ and $f(k)$ is estimated by two distributed estimators, with $C_{p i}=\left[\begin{array}{ll}1 & 0\end{array}\right],(p, i=1,2), D_{11}=0.1$,

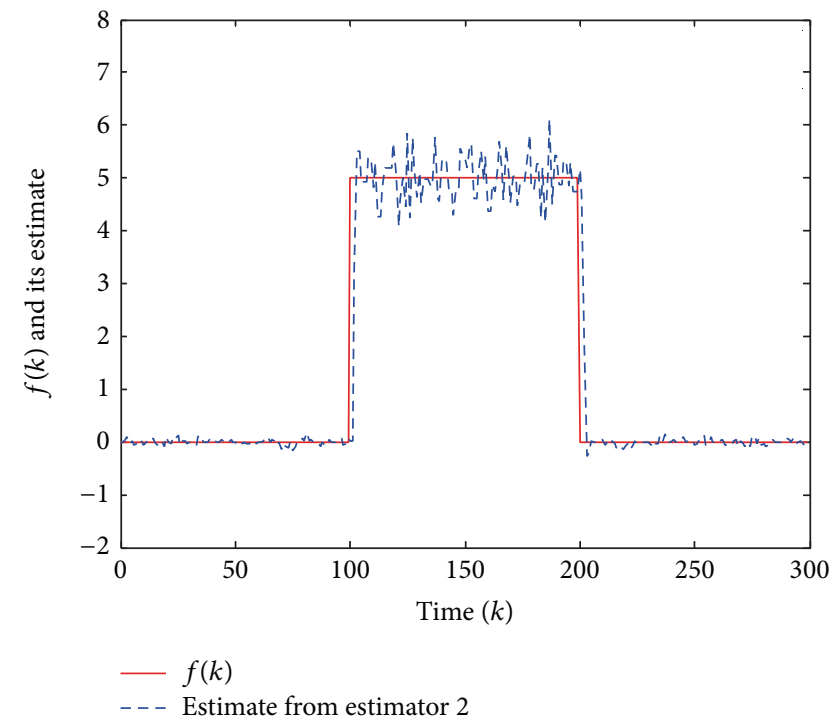

FIGURE 2: $f(k)$ and its estimate $f_{2}(k)$.

$D_{12}=0.1, D_{21}=0.3$, and $D_{22}=0.3$. In order to achieve a better estimation performance, these two estimators share their measurements to each other. This is reasonable as two sensors may be enough to monitor the robotic manipulator and the sensors are usually deployed in the working area of this manipulator such that they can communicate with each other. By choosing $\rho_{1}=0.9, \rho_{2}=0.7$, solving the optimization problem (30), the minimized value for $H_{\infty}$ performance is obtained as $\gamma^{*}=3.2053$ and the corresponding filter parameter matrices are given as follows:

$$
\begin{aligned}
& \bar{H}_{1}=\left[\begin{array}{cc}
1.3854 & -0.1770 \\
0.4810 & 0.2116 \\
1.3770 & -0.1739 \\
0.4969 & 0.2057
\end{array}\right], \\
& \bar{H}_{2}=\left[\begin{array}{cc}
1.3907 & -0.1772 \\
0.4937 & 0.2117 \\
1.3825 & -0.1741 \\
0.5095 & 0.2059
\end{array}\right], \\
& \bar{K}_{1}=\left[\begin{array}{ll}
14.7365 & -3.3897 \\
14.7365 & -3.3897
\end{array}\right], \\
& \bar{K}_{2}=\left[\begin{array}{ll}
14.7305 & -3.3878 \\
14.7305 & -3.3878
\end{array}\right] .
\end{aligned}
$$

For simulation, the disturbance $w(k)$ is randomly varying in $[-0.5,0.5]$ and $f(k)$ is chosen as

$$
f(k)= \begin{cases}0 & 0 \leq k<100 ; 200 \leq k<300 \\ 5 & 100 \leq k<200 ; 300 \leq k<400 .\end{cases}
$$

Simulation results are depicted as follows. The state trajectories of $f(k)$ as well as its estimates $f_{1}(k)$ and $f_{2}(k)$ are shown in Figures 1 and 2, respectively. It follows from 
TABLE 1: Relation between the estimation performance and the quantization density.

\begin{tabular}{cccccc}
\hline$\rho_{1}$ & 0.9 & 0.9 & 0.9 & 0.9 & 0.9 \\
\hline$\rho_{2}$ & 0.9 & 0.8 & 0.7 & 0.6 & 0.5 \\
$\gamma^{*}$ & 3.1971 & 3.2053 & 3.2231 & 3.2587 & 3.3578 \\
\hline
\end{tabular}

Figures 1 and 2 that the two sensors have the same estimation performance due the the consensus based estimation technique. Hence, once one sensor has temporary failure such that it cannot provide the estimation signal, we can also have the fault estimation signal from the other sensor. The relation between the quantization density and the estimation performance is given in Table 1 . It is seen that the estimation performance becomes worse when the quantization density is small; however more energy may be saved as less information is transmitted.

\section{Conclusions}

We have investigated the distributed fault estimation for a class of nonlinear systems, where the nonlinear plant is approximated by a T-S fuzzy model. Due to the limited power in sensors, signal quantization technique has been utilized to save sensor power. Based on the Lyapunov stability theory and the robust control approach, a sufficient condition is obtained such that the estimation error system is asymptotic stable with a prescribed $H_{\infty}$ performance level. Finally, a case study on the fault estimation of actuator fault in the robotic manipulator is given to show the effectiveness of the proposed design. Some other future works, for example, the distributed fault estimation, with various networked issues will be considered [15-18]. In addition, in order to reduce the energy consumption of network, stochastic transmission protocol may be employed [19]. In this scenario, how to design the distributed fault estimator deserves further study.

\section{Conflict of Interests}

The authors declare that there is no conflict of interests regarding the publication of this paper.

\section{Acknowledgments}

This work was supported by the Zhejiang Provincial Natural Science Foundation of China (no. LQ14F030002) and also by the National Key Technology R\&D Program (no. 2012BAH38F01-02).

\section{References}

[1] R. J. Patton and J. Chen, "Robust fault detection using eigenstructure assignment: a tutorial consideration and some new results," in Proceedings of the 30th IEEE Conference on Decision and Control, pp. 2242-2247, Brighton, UK, December 1991.
[2] R. J. Patton and J. Chen, "Review of parity space approaches to fault diagnosis for aerospace systems," Journal of Guidance, Control, and Dynamics, vol. 17, no. 2, pp. 278-285, 1994.

[3] R. K. Mehra and J. Peschon, "An innovations approach to fault detection and diagnosis in dynamic systems," Automatica, vol. 7, no. 5, pp. 637-640, 1971.

[4] I. Hwang, S. Kim, Y. Kim, and C. E. Seah, "A survey of fault detection, isolation, and reconfiguration methods," IEEE Transactions on Control Systems Technology, vol. 18, no. 3, pp. 636-653, 2010

[5] S. X. Ding, Model-Based Fault Diagnosis Techniques: Design Schemes, Algorithms, and Tools, Springer, Berlin, Germany, 2008.

[6] B. Jiang and F. N. Chowdhury, "Fault estimation and accommodation for linear MIMO discrete-time systems," IEEE Transactions on Control Systems Technology, vol. 13, no. 3, pp. 493-499, 2005.

[7] K. Zhang, B. Jiang, and P. Shi, "Fault estimation observer design for discrete-time takagi-sugeno fuzzy systems based on piecewise lyapunov functions," IEEE Transactions on Fuzzy Systems, vol. 20, no. 1, pp. 192-200, 2012.

[8] R. Olfati-Saber and J. S. Shamma, "Consensus filters for sensor networks and distributed sensor fusion," in Proceedings of the 44th IEEE Conference on Decision and Control, and the European Control Conference (CDC-ECC '05), pp. 6698-6703, Seville, Spain, December 2005.

[9] B. Shen, Z. Wang, and Y. S. Hung, "Distributed $H_{\infty}$-consensus filtering in sensor networks with multiple missing measurements: the finite-horizon case," Automatica, vol. 46, no. 10, pp. $1682-1688,2010$.

[10] D. Zhang, L. Yu, H. Song, and Q.-G. Wang, "Distributed $H_{\infty}$ filtering for sensor networks with switching topology," International Journal of Systems Science, vol. 44, no. 11, pp. 21042118, 2013.

[11] M. Fu and L. Xie, "The sector bound approach to quantized feedback control," IEEE Transactions on Automatic Control, vol. 50, no. 11, pp. 1698-1711, 2005.

[12] J. C. Geromel, M. C. de Oliveira, and J. Bernussou, "Robust filtering of discrete-time linear systems with parameter dependent Lyapunov functions," SIAM Journal on Control and Optimization, vol. 41, no. 3, pp. 700-711, 2002.

[13] Y. J. Pan, H. J. Marquez, and T. Chen, "Sampled-data iterative learning control for a class of nonlinear networked control systems," in Proceedings of the American Control Conference, vol. 1-12, pp. 3494-3499, June 2006.

[14] H. Zhang, J. Yang, and C. Y. Su, "T-S fuzzy-model-based robust $H_{\infty}$ design for networked control systems with uncertainties," IEEE Transactions on Industrial Informatics, vol. 3, no. 4, pp. 289-301, 2007.

[15] H. Shen, S. Xu, J. Lu, and J. Zhou, "Passivity-based control for uncertain stochastic jumping systems with mode-dependent round-trip time delays," Journal of the Franklin Institute, vol. 349, no. 5, pp. 1665-1680, 2012.

[16] H. Shen, J. H. Park, L. X. Zhang, and Z. G. Wu, "Robust extended dissipative control for sampled-data Markov jump systems," International Journal of Control, vol. 87, no. 8, pp. 1549-1564, 2014.

[17] Z. G. Wu, P. Shi, H. Su, and J. Chu, "Asynchronous $l_{2}-l_{\infty}$ filtering for discrete-time stochastic Markov jump systems with 
randomly occurred sensor nonlinearities," Automatica, vol. 50, no. 1, pp. 180-186, 2014.

[18] Y. Tang, H. Gao, and J. Kurths, "Distributed robust synchronization of dynamical networks with stochastic coupling," IEEE Transactions on Circuits and Systems I: Regular Papers, vol. 61, no. 5, pp. 1508-1519, 2014.

[19] C. Zhang, G. Feng, J. Qiu, and W.-A. Zhang, “T-S fuzzymodel-based piecewise $H_{\infty}$ output feedback controller design for networked nonlinear systems with medium access constraint," Fuzzy Sets and Systems, vol. 248, pp. 86-105, 2014. 


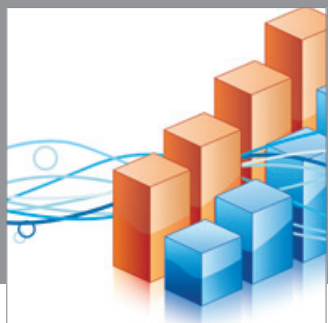

Advances in

Operations Research

mansans

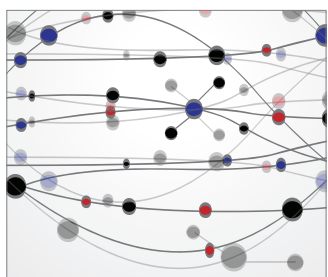

The Scientific World Journal
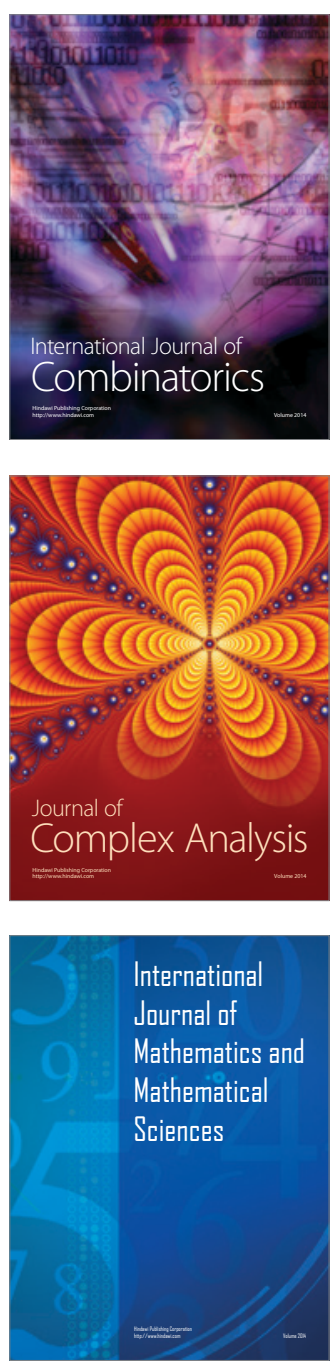
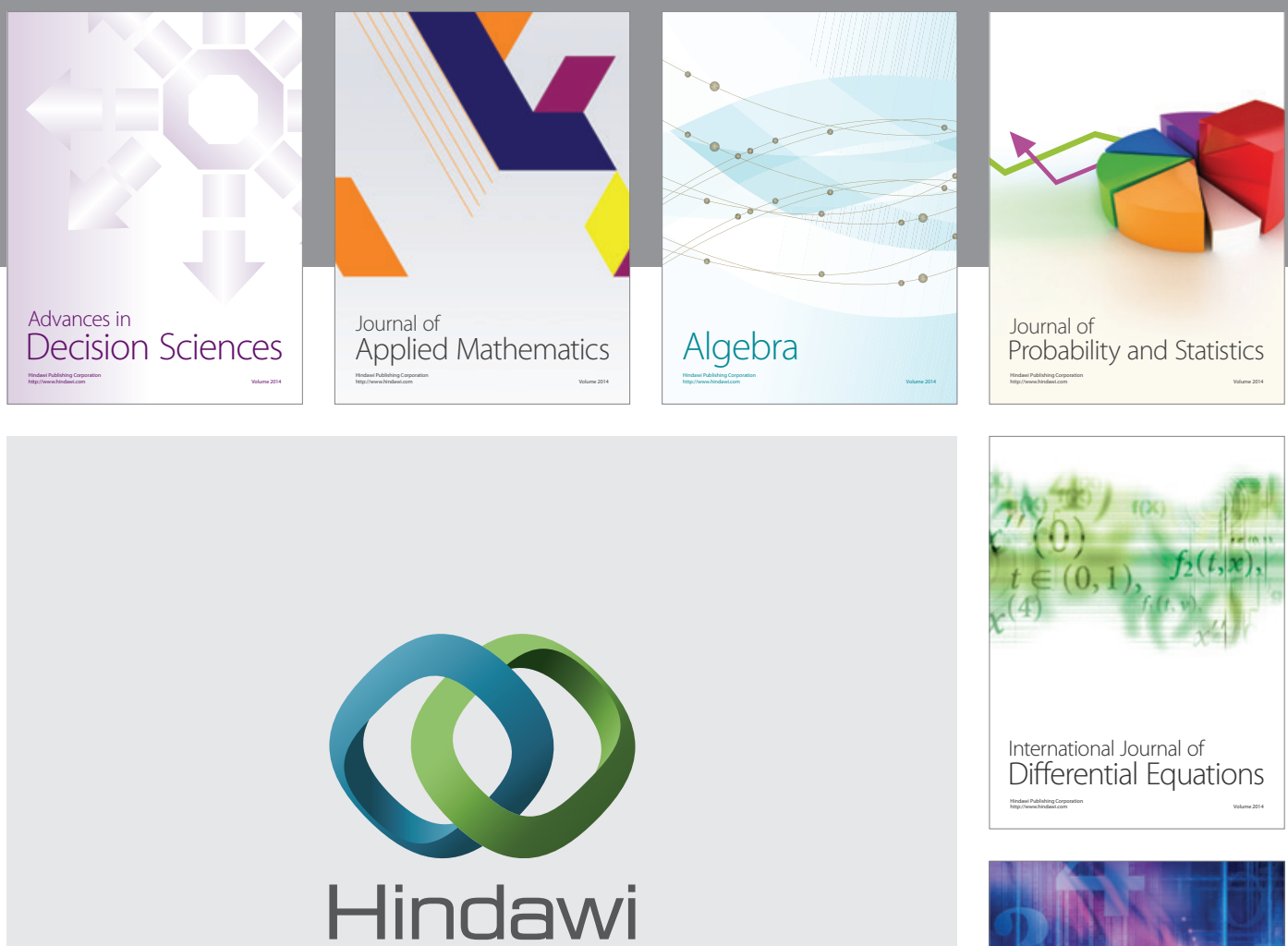

Submit your manuscripts at http://www.hindawi.com
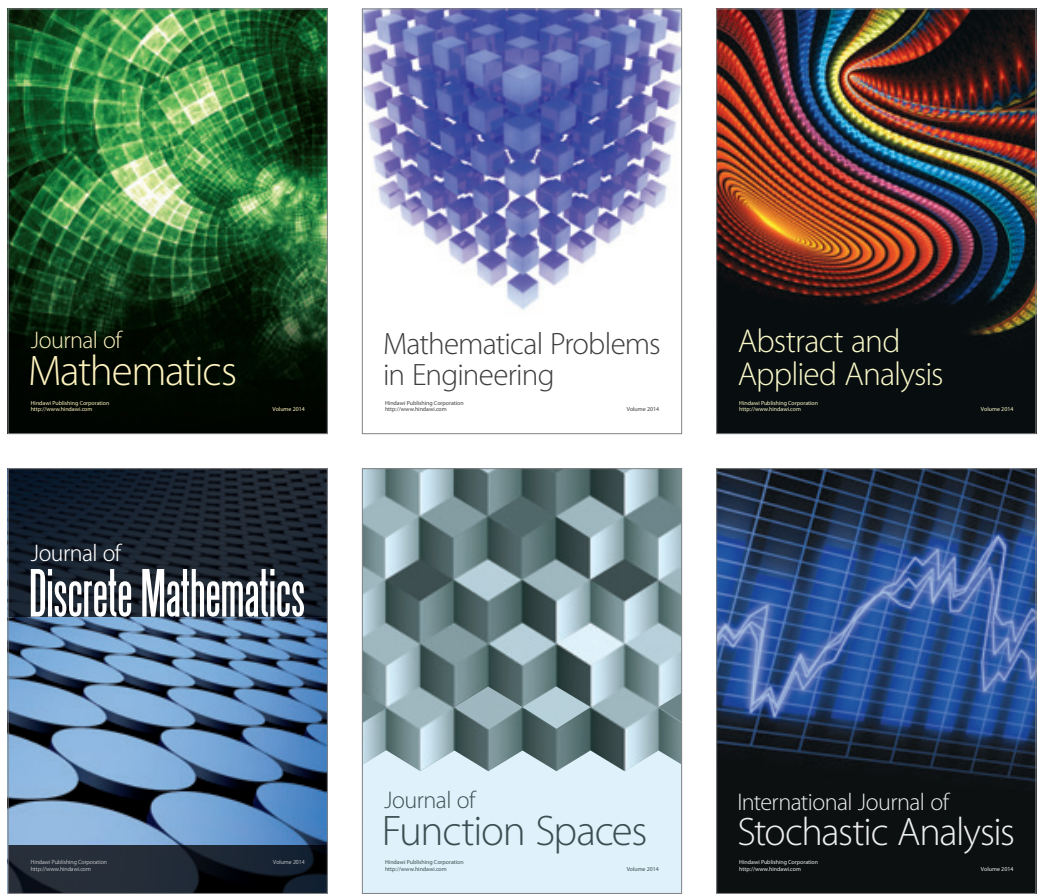

Journal of

Function Spaces

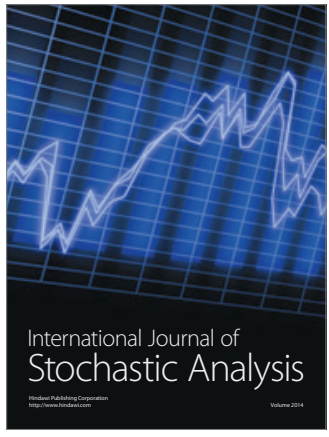

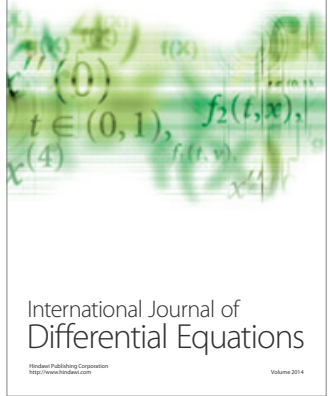
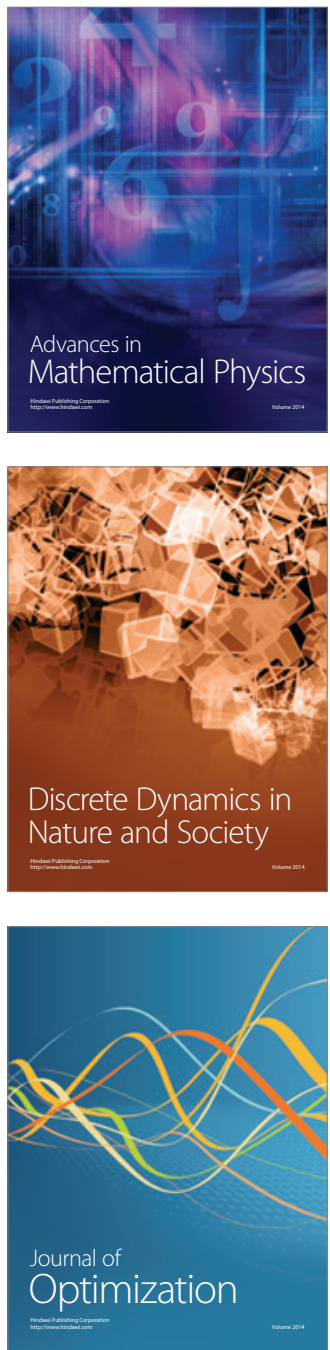